\title{
Wet-Bulb Temperature from Relative Humidity and Air Temperature
}

\author{
ROLAND STULL \\ University of British Columbia, Vancouver, British Columbia, Canada
}

(Manuscript received 14 July 2011, in final form 28 August 2011)

\begin{abstract}
An equation is presented for wet-bulb temperature as a function of air temperature and relative humidity at standard sea level pressure. It was found as an empirical fit using gene-expression programming. This equation is valid for relative humidities between $5 \%$ and $99 \%$ and for air temperatures between $-20^{\circ}$ and $50^{\circ} \mathrm{C}$, except for situations having both low humidity and cold temperature. Over the valid range, errors in wet-bulb temperature range from $-1^{\circ}$ to $+0.65^{\circ} \mathrm{C}$, with mean absolute error of less than $0.3^{\circ} \mathrm{C}$.
\end{abstract}

\section{Introduction}

To calculate relative humidity in percent (hereinafter denoted as RH\%) from dry-bulb temperature $T$ and wetbulb temperature $T_{w}$, one can use a well-known set of "forward" analytical psychrometric equations (Bohren and Albrecht 1998; Stull 2011). For any pressure, such as standard sea level pressure of $101.325 \mathrm{kPa}$, the resulting calculated values of $\mathrm{RH} \%$ can be listed in psychrometric tables or plotted in graphs such as Fig. 1. There is, however, no easy analytical "inverse" solution to get $T_{w}$ from $T$ and $\mathrm{RH} \%$.

Yet some applications require estimates of $T_{w}$, given electronic sensor measurements or numerical forecasts of $T$ and $\mathrm{RH} \%$. For example, the "wet-bulb globe temperature" is used by industrial hygienists, athletes, and the military to estimate the composite effect of temperature, solar radiation, humidity, and wind speed on people (see, e.g., online at http://en.wikipedia.org/wiki/ Wet_Bulb_Globe_Temperature). The effectiveness of snowmaking equipment is a strong function of $T_{w}$, because snow can be produced in air temperatures slightly above freezing if $T_{w}$ is below about $-2^{\circ} \mathrm{C}$ (see, e.g., online at http://en.wikipedia.org/wiki/Snowmaking). In a similar way, subzero $T_{w}$ values can exacerbate freezing-rain events. Lower $T_{w}$ allows industrial cooling towers and other evaporative coolers (also called swamp coolers or desert coolers) to operate more efficiently (see http:// en.wikipedia.org/wiki/Wet-bulb_temperature; http:// en.wikipedia.org/wiki/Evaporative_cooler). Wet-bulb temperature $T_{w}$ and wet-bulb potential temperature can be used to label saturated adiabats on thermodynamic diagrams such as skew Ts and tephigrams (Stull 2011). In a similar way, sometimes it is desirable to estimate $T_{w}$ without using Normand's rule (Stull 2011) on a thermodynamic diagram.

\section{Empirical expression for wet-bulb temperature}

Presented here is an empirical inverse solution found by a function fit to the data in Fig. 1 . It yields $T_{w}\left({ }^{\circ} \mathrm{C}\right)$ as a function of $T\left({ }^{\circ} \mathrm{C}\right)$ and $\mathrm{RH} \%$ (where a humidity such as $65.8 \%$ is input as the number 65.8$)$ :

$$
\begin{aligned}
T_{w}= & T \operatorname{atan}\left[0.151977(\mathrm{RH} \%+8.313659)^{1 / 2}\right]+\operatorname{atan}(T+\mathrm{RH} \%)-\operatorname{atan}(\mathrm{RH} \%-1.676331) \\
& +0.00391838(\mathrm{RH} \%)^{3 / 2} \operatorname{atan}(0.023101 \mathrm{RH} \%)-4.686035 .
\end{aligned}
$$

Corresponding author address: Roland Stull, Earth and Ocean Sciences Dept., University of British Columbia, 6339 Stores Rd., Vancouver, BC V6T 1Z4, Canada.

E-mail: rstull@eos.ubc.ca
The arctangent function uses argument values as if they are in radians. The curves in Fig. 2 were calculated using Eq. (1) to show $T_{w}$ as an explicit function of $T$ and RH\%. Equation (1) is valid for a pressure of $101.325 \mathrm{kPa}$ and for the combinations of dry-bulb temperatures and 
relative humidities as plotted in Fig. 2. Saturation is with respect to liquid water over all temperatures.
To illustrate its usage, plugging $T\left({ }^{\circ} \mathrm{C}\right)=20$ and $\mathrm{RH} \%=$ 50 into Eq. (1) gives

$$
\begin{aligned}
T_{w}= & 20 \operatorname{atan}\left[0.151977(50+8.313659)^{1 / 2}\right]+\operatorname{atan}(20+50)-\operatorname{atan}(50-1.676331) \\
& +0.00391838(50)^{3 / 2} \operatorname{atan}(0.023101 \times 50)-4.686035=13.7^{\circ} \mathrm{C} .
\end{aligned}
$$

The errors between the Eq. (1) estimate and the $T_{w}$ values from Fig. 1 are plotted in Fig. 3. Mean error is $-0.0052^{\circ} \mathrm{C}$, median error is $0.026^{\circ} \mathrm{C}$, mean absolute error is $0.28^{\circ} \mathrm{C}$, and the fraction of variance $\left(r^{2}\right)$ explained by the regression is $99.95 \%$.

Figures 2 and 3 are plotted on the same scale so that at any point ( $T, \mathrm{RH} \%)$ one can find $T_{w}$ from Fig. 2 and the corresponding error from Fig. 3. Two factors influence the errors in Fig. 3. One is that the regression was based only on the data from Fig. 1, for $1 \% \leq \mathrm{RH} \% \leq 99 \%$. Thus, the region of cold temperatures and low relative humidities in the lower-right corner of Fig. 1, for which no data are provided, corresponds to the region in the lower-left corner of Fig. 3, for which the regression does not apply. The valid region from Fig. 3 is outlined with the dashed line in Fig. 2. The second factor is that the regression is highly nonlinear and is not based on physical principles. Thus, the $T_{w}$ errors have multiple relative maxima and minima of order from $-1.0^{\circ}$ to $+0.6^{\circ} \mathrm{C}$, with larger errors generally near the edges of the valid domain. Outside the valid region plotted in Figs. 2 and 3, $T_{w}$ from the regression equation can quickly diverge from reality.

\section{Regression method}

Gene-expression programming (GEP) was the method used to find a best-fit function to the data. GEP (Ferreira 2006) is an efficient variant of genetic programming, in which candidate functions evolve through various forms of mutation and compete by a computational natural selection until the fittest candidate (with the lowest verification error) is found. Details of GEP for meteorological applications are presented by Bakhshaii and Stull (2009) and Roebber (2010). GEP can explore a wide range of the function space to find a best fit and can yield a nonlinear result [such as Eq. (1)] that would not necessarily have been obvious if the function fit had been attempted manually.

The input data for this regression were only $T_{w}, T$, and RH\% for standard sea level pressure of $101.325 \mathrm{kPa}$. Pressure was not used as one of the predictor variables, and hence the resulting regression does not vary with pressure. Also, because the regression equation is only a statistical fit and is not based on physical principles, one cannot assume that it would be valid at other pressures. If one wanted to apply Eq. (1) to other pressures, however, how much error can one expect? The error is indicated in Fig. 2 by the difference between the thick black curve [from Eq. (1)] and the light-gray curves as found from traditional forward calculations.

As an alternative, the reader can use GEP to create new regressions based on $T_{w}, T$, and $\mathrm{RH} \%$ data for any other pressure of interest. Given the quasi-random nature of evolution, it is unlikely that the resulting equations would look like Eq. (1). Namely, both the functional form and the numerical coefficients would likely be considerably different.

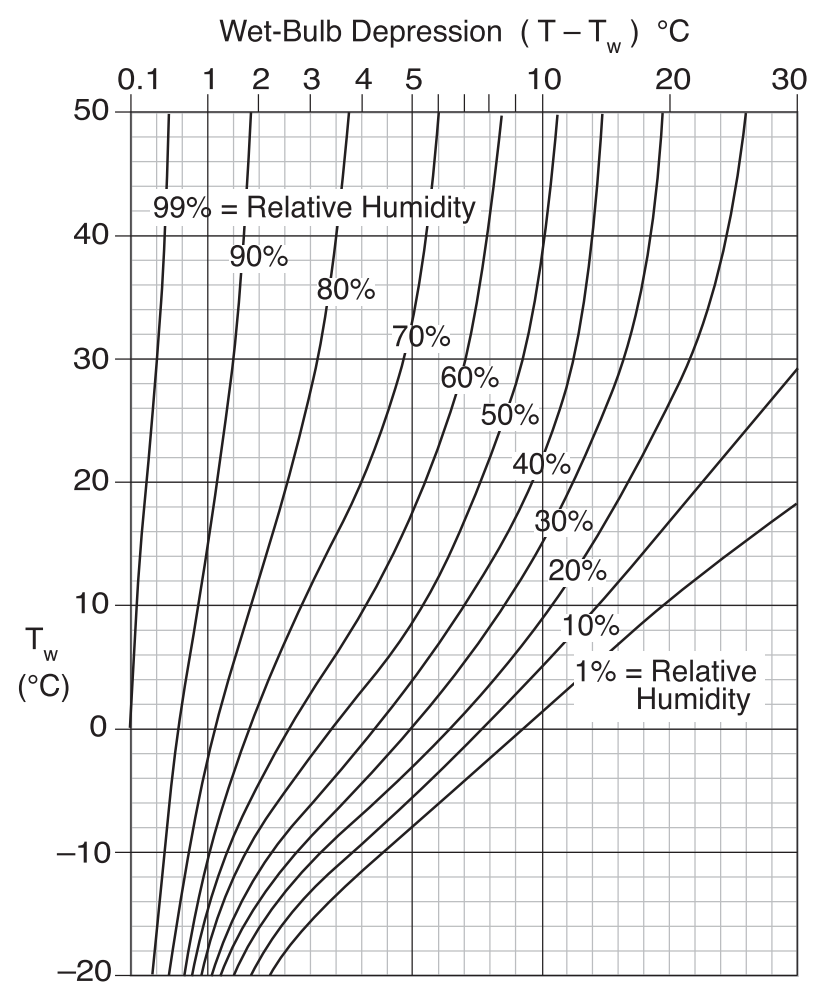

FIG. 1. Psychrometric graph for standard sea level pressure of $101.325 \mathrm{kPa}$. The abscissa changes scale at the dark vertical lines. In the saturation calculation to determine relative humidity, Teten's equation was used to account for variations in latent heat of vaporization (Stull 2011). 


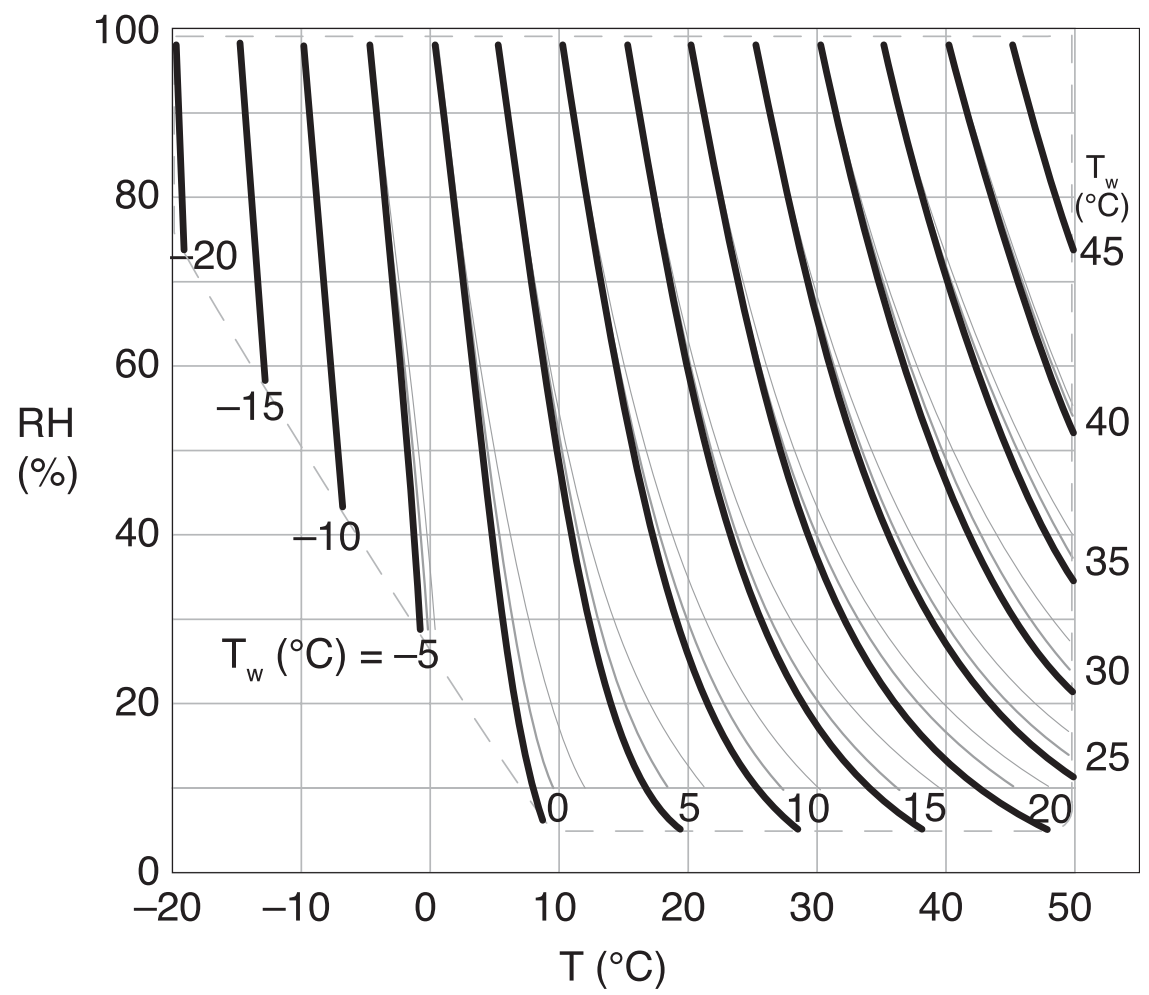

FIG. 2. Isopleths of $T_{w}$ (thick black curves) vs RH\% and $T$, found from Eq. (1). The valid range is enclosed by a dashed line, and the valid pressure is $101.325 \mathrm{kPa}$. The gray curves associated with each $T_{w}$ are for $P=80 \mathrm{kPa}$ (thinner lines) and $P=60 \mathrm{kPa}$ (thinnest lines, located farther away from each black line). These gray curves [not found from Eq. (1)] are useful for estimating the error if Eq. (1) is applied to pressures that are not equal to $101.325 \mathrm{kPa}$.

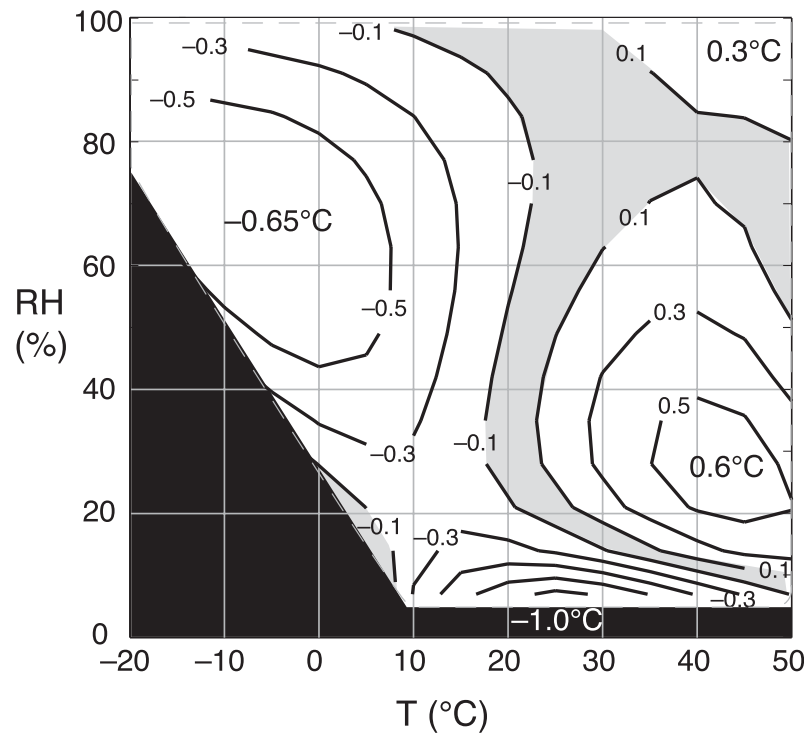

FIG. 3. The $T_{w}$ errors $\left({ }^{\circ} \mathrm{C}\right)$, computed as Eq. (1) values minus the values from Fig. 1, for $P=101.325 \mathrm{kPa}$. Gray shading highlights nearzero errors. The empirical fit is not valid in the black-shaded region.
Acknowledgments. I am grateful to Atoossa Bakhshaii who helped with the graphics. This research was supported with a grant from the Canadian Natural Sciences and Engineering Research Council.

\section{REFERENCES}

Bakhshaii, A., and R. Stull, 2009: Deterministic ensemble forecasts using gene-expression programming. Wea. Forecasting, 24, 1431-1451.

Bohren, C. F., and B. A. Albrecht, 1998: Atmospheric Thermodynamics. Oxford University Press, 402 pp.

Ferreira, C., 2006: Gene Expression Programming: Mathematical Modeling by an Artificial Intelligence. 2nd ed. Springer, 478 pp.

Roebber, P. J., 2010: Seeking consensus: A new approach. Mon. Wea. Rev., 138, 4402-4415.

Stull, R., 2011: Meteorology for Scientists and Engineers. 3rd ed. Discount Textbooks, 924 pp. [Available online at http://www. eos.ubc.ca/courses/atsc201/MSE3.html.] 